\title{
Differential mRNA Expression Levels of Human Histone-Modifying Enzymes in Normal Karyotype B Cell Pediatric Acute Lymphoblastic Leukemia
}

\section{Yan-Fang Tao ${ }^{1}$, Li Pang ${ }^{1}$, Xiao-Juan Du ${ }^{2}$, Li-Chao Sun ${ }^{3}$, Shao-Yan Hu ${ }^{1}$, Jun Lu ${ }^{1}$, Lan Cao ${ }^{1}$,} Wen-Li Zhao ${ }^{1}$, Xing Feng ${ }^{1}$, Jian Wang ${ }^{1}$, Dong Wu ${ }^{1}$, Na Wang ${ }^{1}$, Jian Ni ${ }^{4}$ and Jian Pan ${ }^{1, *}$

1 Department of Hematology and Oncology, Children's Hospital of Soochow University, Suzhou 215003, Jiangsu, China; E-Mails: taoyanfang1982@163.com (Y.-F.T.); pangyeli2011@163.com (L.P.); hsy139@126.com (S.-Y.H.); lujun_sz@yahoo.com.cn (J.L.); c12012@sohu.com (L.C.); zhaowenli69@yahoo.com.cn (W.-L.Z.); xing_feng66@hotmail.com (X.F.);wj196312@vip.163.com (J.W.); wudong.1987@163.com (D.W.); ontheway120@126.com (N.W.)

Department of Gastroenterology, the 5th Hospital of Chinese PLA, Yinchuan 750004, Ningxia, China; E-Mail: du_xiaojuan123@163.com

3 Department of Cell and Molecular Biology, Cancer Institute (Hospital), Chinese Academy of Medical Sciences, Peking Union Medical College, Beijing100021, China; E-Mail: sunlichao_1980@hotmail.com

Translational Research Center, The Second Clinical School, Nanjing Medical University, Nanjing 210011, Jiangsu, China; E-Mail: ni_jian2008@163.com

* Author to whom correspondence should be addressed; E-Mail: panjian2008@163.com; Tel./Fax: +86-512-6778-8216.

Received: 21 November 2012; in revised form: 29 January 2013 / Accepted: 30 January 2013 / Published: 6 February 2013

\begin{abstract}
Histone modification enzymes regulate gene expression by altering the accessibility of promoters to transcription factors. We sought to determine whether the genes encoding histone modification enzymes are dysregulated in pediatric acute lymphoblastic leukemia (ALL). A real-time PCR array was designed, tested and used to profile the expression of 85 genes encoding histone modification enzymes in bone marrow mononuclear cells from 30 pediatric ALL patients and 20 normal controls. The expression profile of histone-modifying genes was significantly different between normal karyotype B cell pediatric ALL and normal controls. Eleven genes were upregulated in pediatric ALL, including the histone deacetylases $H D A C 2$ and $P A K 1$, and seven genes were downregulated,
\end{abstract}


including PRMT2 and the putative tumor suppressor EP300. Future studies will seek to determine whether these genes serve as biomarkers of pediatric ALL. Ingenuity Pathway Analysis revealed that Gene Expression and Organ Morphology was the highest rated network, with 13 focus molecules (significance score $=35$ ). Ingenuity Pathway Analysis also indicated that curcumin and miR-34 are upstream regulators of histone-modifying enzymes; future studies will seek to validate these results and examine the role of curcumin and miR-34 in leukemia. This study provides new clues into the molecular mechanisms of pediatric ALL.

Keywords: histone-modifying enzymes; pediatric acute lymphoblastic leukemia; real-time PCR array

\section{Introduction}

Acute lymphoblastic leukemia (ALL) is the most common malignancy diagnosed in children, representing nearly one third of all pediatric cancers [1-4]. Over the last decade, advances in the treatment of pediatric ALL have led to long-term event-free survival rates of approximately $80 \%$. Despite the good overall prognosis, some of the less common subtypes of ALL have a high risk of relapse [1-4]. Rearrangements of the myeloid/lymphoid or mixed-lineage leukemia $(M L L)$ gene at chromosome band 11q23 are detected in least $10 \%$ of cases and are associated with aggressive pediatric ALL. Unfortunately, young children with this genetic abnormality have a very poor prognosis and a survival rate of less than $20 \%$, even after intensive therapy [5]. The $M L L$ gene encodes a DNA-binding protein which methylates histone $\mathrm{H} 3$ lysine 4 (H3K4). MLL is a member of the group of histone-modifying enzymes, which is commonly disrupted in leukemia [6,7]. MLL translocations encode MLL fusion proteins which lack H3K4 methyltransferase activity, which results in abnormal histone modification $[8,9]$.

Histone-modification provides an important regulatory platform for processes such as gene expression, DNA replication and repair, chromosome condensation and segregation and apoptosis. Disruption of these processes has been linked to the multistep process of carcinogenesis [10]. Alterations in histone-modifying enzymes can contribute to the development of a variety of human cancers. The new terminology "histone onco-modifications" has been proposed to describe the post-translational histone modifications linked to cancer [11,12]. Histones are the chief protein components of chromatin, acting as the spools around which DNA winds. Histones are no longer considered to be simple "DNA packaging" proteins, and are currently recognized to be regulators of chromatin dynamics. Histones are subject to a wide variety of post-translational modifications, including acetylation of lysines, methylation of lysines and arginines, serine and threonine phosphorylation, lysine ubiquitylation, glycosylation, sumoylation, adenosine diphosphate ribosylation and carbonylation, all of which are dynamically catalyzed by histone-modifying enzyme complexes [13,14]. Histone modifications influence chromatin-templated processes such as gene transcription, DNA repair and recombination. Histone lysine methylation and acetylation are enzymatically reversible processes which are "written" by lysine methyltransferases (KMTs) [15] and 
lysine acetyltransferases (KATs) [16], and "erased" by lysine demethylases (KDMs) [17-21] and histone deacetylases (HDACs) [22]. Overall, post-translational histone modifications provide an epigenetic mechanism for the regulation of a variety of normal and cancer-related processes. Growing evidence suggests that histone-modifying enzymes are dysregulated in human cancer. In fact, an extensive analysis of the expression patterns of histone-modifying enzymes could discriminate between tumor samples and their normal counterparts, and also cluster the tumor samples according to cell type [10]. However, little is currently known about the histone modification changes which occur during the development and progression of pediatric ALL.

Real-time PCR array systems are an ideal tool for analyzing the expression of a focused panel of genes [23]. The specificity of real-time PCR guarantees the amplification of a single gene-specific product in each reaction, allowing the expression level results to confidently reflect only the gene of interest. PCR arrays can determine the gene expression differences between two RNA samples, with results that are highly concordant with other quantitative gene expression analysis and microarray platforms. PCR arrays also deliver results comparable to high-density microarrays, as well as TaqMan Gene Expression Assays, a widely accepted method for validating the results of microarrays and other more complicated and expensive quantitative methods based on TaqMan assays [24]. In this study, we sought to analyze the mRNA expression profiles of histone-modifying enzymes in pediatric ALL using a powerful real-time PCR array platform.

\section{Results and Discussion}

\subsection{Real-Time PCR Array Design}

We designed and tested 88 real-time PCR primer pairs for quantitative gene expression analysis of genes involved in pediatric ALL. The primers for the target genes are listed in Supplementary File 1. Real-time PCR primers for histone-modifying enzymes. The human histone-modifying enzymes PCR array was designed to profile the expression of 85 key genes, which encode enzymes known or predicted to modify genomic DNA or histones to regulate chromatin accessibility, and therefore gene expression. De novo and maintenance DNA methyltransferases, and the enzymes responsible for the demethylation of $\mathrm{CpG}$ dinucleotides were represented on the array, NOTCH signaling, (NOTCH1, NOTCH2, EP300) and DNA methyltransferases (DNMT1, DNMT3A, DNMT3B). Enzymes catalyzing histone acetylation, methylation, phosphorylation, and ubiquitination were also included on the array, as well as deacetylases and demethylases. The genes included were the histone acetyltransferases: ATF2, CDYL, CIITA, CSRP2BP, ESCO1, ESCO2, HAT1, KAT2A (GCN5L2), KAT2B (PCAF), KAT5 (HTATIP), KAT8, KAT7, KAT6A, KAT6B, NCOA1, NCOA3, NCOA6; histone methyltransferases: CARM1 (PRMT4), DOT1L, EHMT2, MLL, MLL3, PRMT1, PRMT2, PRMT3, PRMT5, PRMT6, PRMT7, PRMT8, SETDB2, SMYD3; enzymes of histone phosphorylation: AURKA, AURKB, AURKC, NEK6, PAK1, RPS6KA3, RPS6KA5; enzymes of histone ubiquitination: DZIP3, RNF2, RNF20, UBE2A, UBE2B, USP16, USP21, USP22; DNA/histone demethylases: KDM1A, KDM5B, KDM5C, KDM4A, KDM4C, MBD2; and histone deacetylases: HDAC1, HDAC2, HDAC3, HDAC4, HDAC5, HDAC6, HDAC7, HDAC8, HDAC9, HDAC10, HDAC11. The array also included genes encoding Drosophila [Su(var), E(z) and Trithorax] (SET) domain proteins, all of which contain a homologous 
domain that confers histone methyltransferase activity in some family members, SET Domain Proteins (Histone Methyltransferase Activity): ASH1L, MLL3, MLL5, NSD1, SETD1A, SETD2, SETD3, SETD4, SETD6, SETD7, SETD8, SETDB1, SUV39H1, SUV420H1, WHSC1.

\subsection{Real-Time PCR Array Testing}

Using real-time PCR, we can easily and reliably analyze the expression of a focused panel of genes involved in epigenetic chromatin modifications with this array. Each primer set was tested by expression analysis and melting curve analysis, to confirm that the primers were specific for the target gene (Figure 1). The flexibility, simplicity and convenience of standard SYBR Green PCR detection methodology makes PCR array systems accessible for routine use in any research laboratory.

Figure 1. Design and testing of the real-time PCR array for human genes encoding epigenetic chromatin modification enzymes. (A) Amplification of a target gene and GAPDH in the real-time PCR array. Reactions were run on a Light cycler 480 (Roche, Basel, Switzerland) using universal thermal cycling parameters $\left(95^{\circ} \mathrm{C}\right.$ for $5 \mathrm{~min}, 45$ cycles of $10 \mathrm{~s}$ at $95{ }^{\circ} \mathrm{C}, 20 \mathrm{~s}$ at $60{ }^{\circ} \mathrm{C}$ and $15 \mathrm{~s}$ at $72{ }^{\circ} \mathrm{C}$ ); (B) Melting curve analysis of the PCR product of a single target gene. Melting curves were generated using the parameters $10 \mathrm{~s}$ at $95{ }^{\circ} \mathrm{C}, 60 \mathrm{~s}$ at $60{ }^{\circ} \mathrm{C}$, followed by continued melting; (C) Amplification of all of the genes in the PCR array.
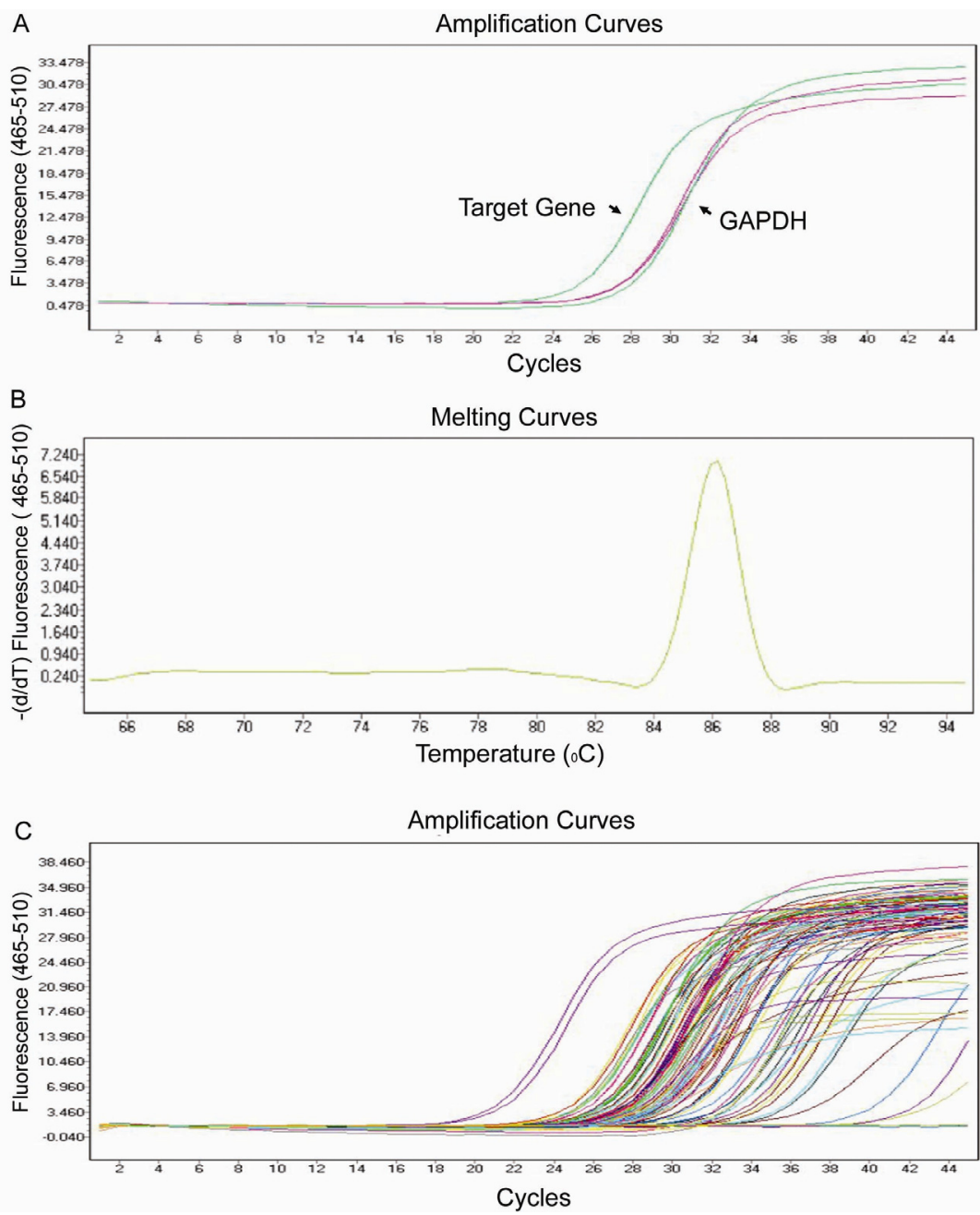


\subsection{Expression Profiling of Normal Karyotype B Cell Pediatric ALL and Normal Control Samples}

We analyzed and clustered the gene expression profiles of bone marrow mononuclear cells from 30 pediatric ALL patients and 20 control samples using the real-time PCR array. The clinical features of the 30 pediatric ALL patients and 20 controls are listed in Table 1. We analyzed the original expression data using Multi Experiment View (MEV) clustering software. The cluster is not successful. This result showed pediatric ALL sample L2, L9, L28, L4, L16, L24, L11, L22, L27, L29 and L30 are different from other ALL samples. L2 and L9 are T cell ALL. L4 and L16 are B cell ALL with MLL fusion gene (Supplementary File 2. Cluster analysis the gene expression in 30 pediatric ALL patients and 20 controls samples). Heterogeneous genetic background may affect the expression of histone-modifying enzymes. So we do the second cluster, and we only clustered gene expression profiles from 18 normal karyotype B cell pediatric ALL patients and 20 control samples The gene expression profile in pediatric ALL was significantly different to the normal controls. Specific sets of genes clustered in normal karyotype B cell ALL (Figure 2). The most significantly clustered genes are shown in Figure 3A. The expression of PAK1 and HDAC2 between normal karyotype B cell ALL and normal control was certificated with western-blot (Figure 3B).

Table 1. Clinical features of the normal donors and pediatric acute lymphoblastic leukemia patients.

\begin{tabular}{|c|c|c|c|}
\hline & & NBM/ITP & Pediatric ALL \\
\hline \multicolumn{2}{|l|}{ Age } & $4.3(0.7-13.6)$ & $5.1(0.9-13.6)$ \\
\hline \multicolumn{2}{|c|}{$\operatorname{Sex}(\mathrm{M} / \mathrm{F})$} & $12 / 8$ & $19 / 11$ \\
\hline \multicolumn{2}{|c|}{ White blood cells (109/L) } & $8.4(3.82-16.97)$ & $56.9(2.1-638)$ \\
\hline \multicolumn{2}{|c|}{ Hemoglobin $(\mathrm{g} / \mathrm{L})$} & $129(90-157)$ & $81.2(28-126)$ \\
\hline \multicolumn{2}{|c|}{ Platelet count (109/L) } & $313(17-498)$ & $49(8-195)$ \\
\hline \multirow{3}{*}{ Immunophenotyping } & B-ALL & $\mathrm{ns}$ & 28 \\
\hline & T-ALL & $\mathrm{ns}$ & 2 \\
\hline & Standard & $\mathrm{ns}$ & 6 \\
\hline \multirow[t]{2}{*}{ Risk stratification } & Median & ns & 8 \\
\hline & High & ns & 16 \\
\hline \multirow{3}{*}{ Karyotype } & Normal & $\mathrm{ns}$ & 18 \\
\hline & Abnormal & $\mathrm{ns}$ & 12 \\
\hline & MLL & $\mathrm{ns}$ & 2 \\
\hline \multirow[t]{3}{*}{ Fusion gene } & TEL/AML1 & $\mathrm{ns}$ & 7 \\
\hline & BCR/ABL1 & $\mathrm{ns}$ & 1 \\
\hline & $\mathrm{E} 2 \mathrm{~A} / \mathrm{PBX}$ & ns & 1 \\
\hline
\end{tabular}

NBM, Normal Bone Marrow; IPT, Idiopathic Thrombocytopenic Purpura; B-ALL, B cell acute lymphoblastic leukemia; T-ALL, T cell acute lymphoblastic leukemia 
Figure 2. Expression and clustering analysis of differentially expressed genes encoding epigenetic chromatin modification enzymes in pediatric ALL and normal control samples. Clustering analysis of the gene expression data from the real-time PCR array. The comparative $C_{t}$ method was used for quantification of gene expression. The gene expression levels for each target gene were normalized to the housekeeping gene GAPDH within the same sample $\left(-\Delta \mathrm{C}_{\mathrm{t}}\right)$; then the relative expression of each gene $(n=87)$ was calculated using $10^{6} \times \log _{2}\left(-\Delta C_{t}\right)$. Gene expression in the normal karyotype $B$ cell pediatric acute lymphoblastic leukemia (ALL) $(n=18)$ and control samples $(n=20)$ was analyzed using Multi Experiment View (MEV) clustering software.

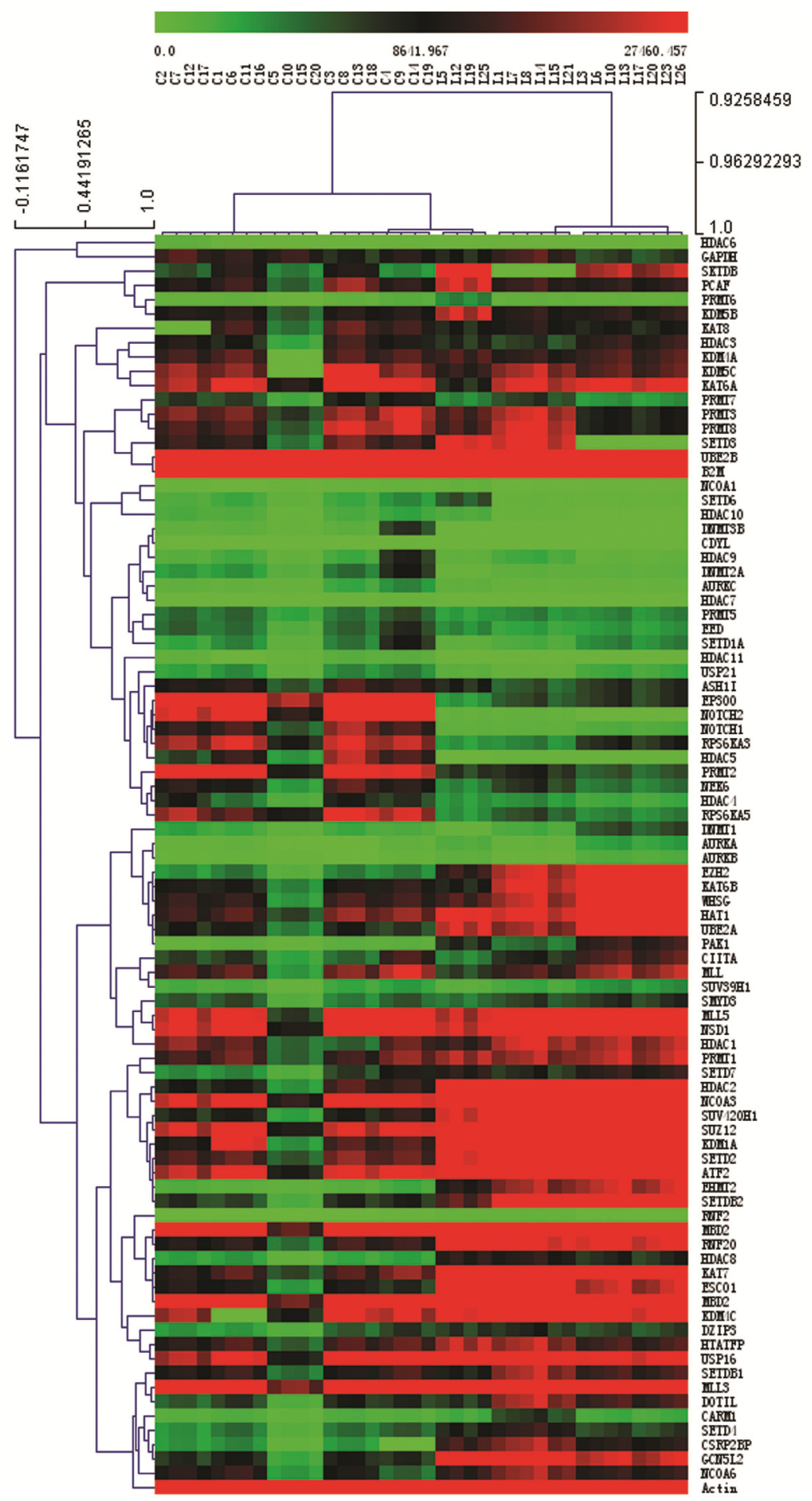


The gene expression profile in pediatric ALL was significantly different to the normal controls. Specific sets of genes clustered in normal karyotype B cell ALL (Figure 2). The most significantly clustered genes are shown in Figure 3A. The expression of PAK1 and HDAC2 between normal karyotype B cell ALL and normal control was certificated with western-blot (Figure 3B).

Figure 3. Expression and clustering analysis of differentially expressed genes encoding epigenetic chromatin modification enzymes in pediatric ALL and normal control samples. (A) The most significantly clustered genes between normal karyotype B cell ALL and normal controls; (B) Western-blot analysis the expression of PAK1 and HDAC2 in pediatric ALL and normal control samples.

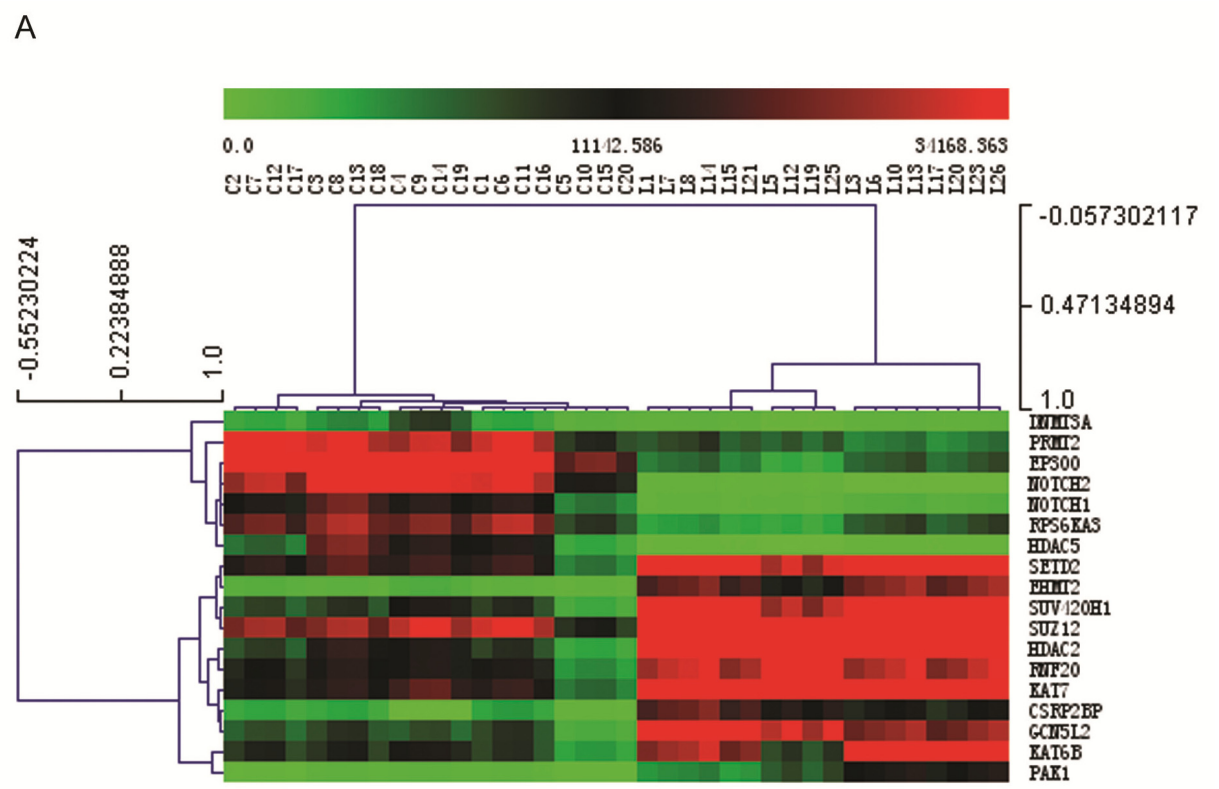

B

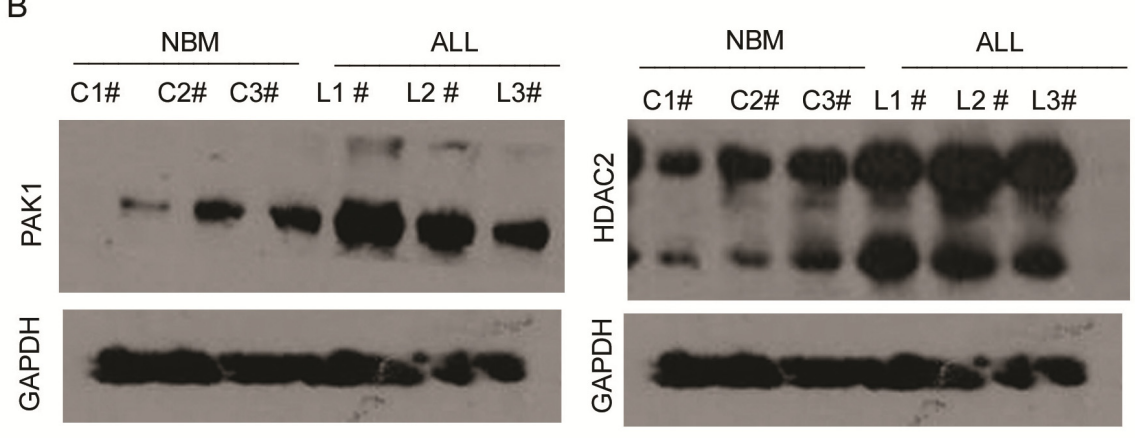

The 11 genes upregulated in normal karyotype B cell pediatric ALL are listed in Table 2 .The expression level of each upregulated gene in pediatric ALL is presented in Figure 4. Some of these upregulated genes have previously been studied in leukemia or other tumors. The gene expression profile of 12 HDAC genes was previously analyzed by quantitative real-time PCR in 94 consecutive cases of childhood ALL [10,25]. The ALL samples showed higher expression levels of HDAC2, compared to normal bone marrow samples [10,25], in agreement with this study. The epigenetic regulator $H D A C 2$ is often significantly overexpressed in solid tumors, can influence cell proliferation, apoptosis and differentiation, and has been suggested as a therapeutically important prognostic marker [26-29]. 
Table 2. Genes encoding epigenetic chromatin modification enzymes upregulated in normal karyotype B cell pediatric ALL compared with normal controls.

\begin{tabular}{ccccccc}
\hline & Gene & Description & NBM & ALL & Change & $\boldsymbol{p}$ value \\
\hline 1 & PAK1 & P21 protein (Cdc42/Rac)-activated kinase 1 & 690.78 & 8684.84 & 12.57 & $3.94 \times 10^{-17}$ \\
2 & EHMT2 & Euchromatic histone-lysine N-methyltransferase 2 & 1238.91 & 19701.33 & 15.90 & $1.07 \times 10^{-15}$ \\
3 & KAT7 & K(lysine) acetyltransferase 7 & 13037.87 & 76644.47 & 5.88 & $5.97 \times 10^{-9}$ \\
4 & GCN5L2 & K(lysine) acetyltransferase 2A & 6554.99 & 33808.15 & 5.16 & $6.03 \times 10^{-9}$ \\
5 & SUZ12 & Suppressor of zeste 12 homolog & 24556.24 & 123398.3 & 5.03 & $1.56 \times 10^{-6}$ \\
6 & SUV420H1 & Suppressor of variegation 4-20 homolog 1 & 7843.03 & 44187.54 & 5.63 & $1.65 \times 10^{-6}$ \\
7 & KAT6B & K(lysine) acetyltransferase 6B & 26130.51 & 75299.71 & 2.88 & $4.19 \times 10^{-6}$ \\
8 & CSRP2BP & CSRP2 binding protein & 2041.52 & 14595.22 & 7.15 & $2.25 \times 10^{-5}$ \\
9 & RNF20 & Ring finger protein 20 & 10498.77 & 32675.14 & 3.11 & 0.00034 \\
10 & SETD2 & SET domain containing 2 & 14027.6 & 42467.29 & 3.02 & 0.008 \\
11 & HDAC2 & Histone deacetylase 2 & 9325.25 & 50147.01 & 5.38 & 0.015 \\
\hline
\end{tabular}

NBM, Normal Bone Marrow.

Figure 4. Expression of upregulated epigenetic chromatin modification genes in normal karyotype B cell pediatric ALL. Expression levels of the significantly upregulated genes in normal karyotype B cell pediatric ALL $(n=18)$, compared to the control samples (NBT/IPT; $n=20)$. Data is presented as the average $\pm \mathrm{SE} ; p$ values $<0.05$ were considered statistically significant.
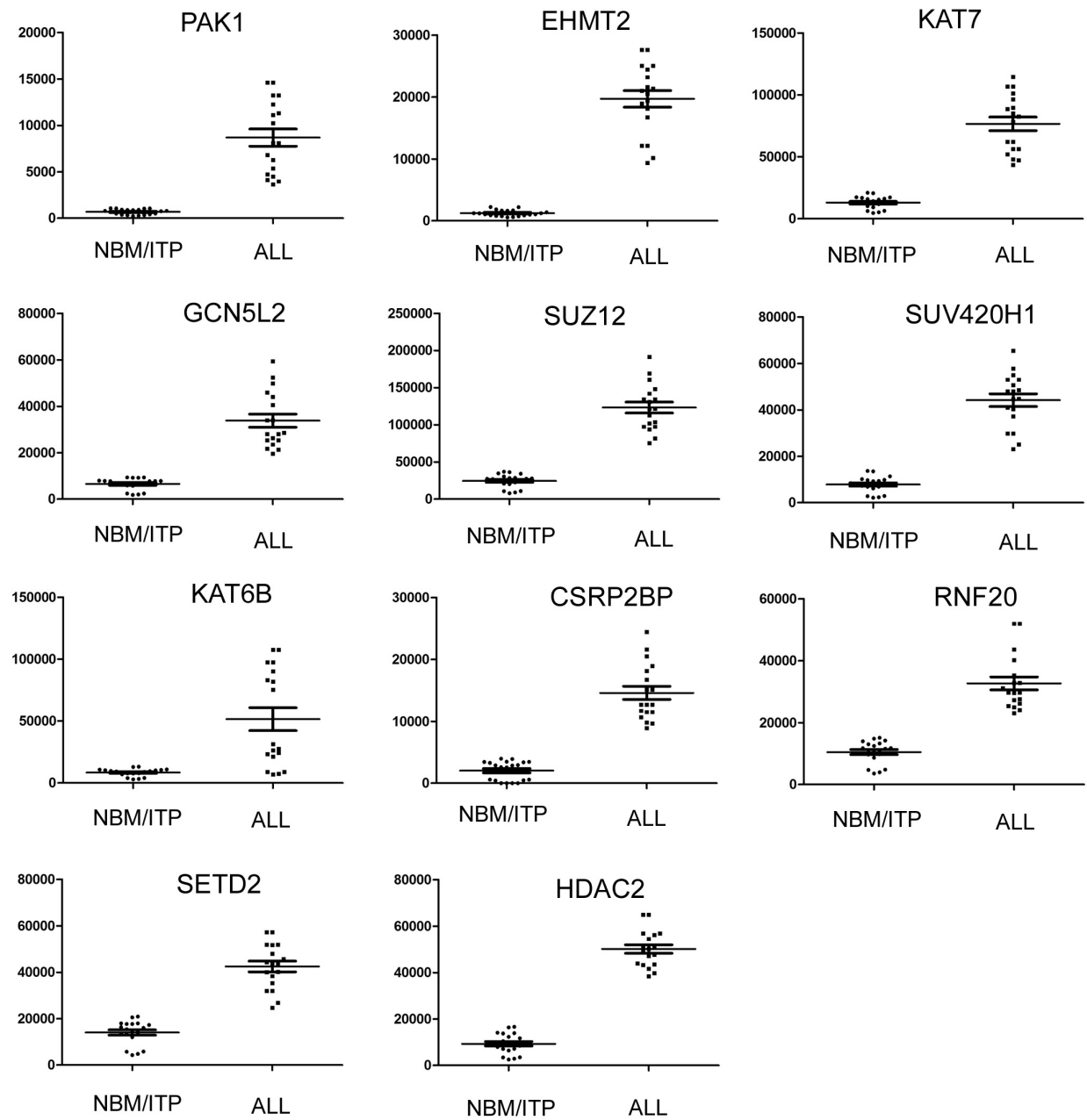
Changes in the levels and activity of p21 protein (Cdc42/Rac)-activated kinase 1 (PAK1) are also frequently described in human malignancies [30-33]. This phenomenon has been observed in various tumor types using a variety of techniques. The abnormalities reported include gene amplification, elevated mRNA and protein expression, and increased accumulation of the phosphorylated and, presumably, activated form of this enzyme. There are also intriguing observations regarding the accumulation of phosphorylated PAK1 specifically in the nuclei of malignant cells [34], which parallel the changes observed during tumor progression in a mouse model [35]. Importantly, elevated levels of PAK1 were identified to be an independent prognostic predictor of poor survival in ovarian cancer [36]. In breast cancer, nuclear expression of PAK1, in conjunction with phosphorylation of the estrogen receptor on the PAK1 site (serine 305), predicts resistance to tamoxifen therapy, and the cytoplasmic levels of PAK1 correlate with the recurrence rate and mortality [35,37]. Similarly, higher levels of PAK1 were associated with advanced tumor stage, metastasis and reduced survival in patients with gastric cancer [38,39]. There are also numerous reports of elevated PAK1 activity in cell lines, although in most of these cases such reports cannot rule out the possibility that the changes have been selected for or caused by in vitro culture.

The seven genes downregulated in pediatric ALL are listed in Table 3, and the expression level of each downregulated gene in pediatric ALL is presented in Figure 5. The putative tumor suppressor gene EP300 is located on chromosome 22q13, a region which shows frequent loss of heterozygosity $(\mathrm{LOH})$ in colon, breast and ovarian cancer. $\mathrm{LOH}$ across the EP300 locus was detected in $38 \%$ of colon, $36 \%$ of breast, and $49 \%$ of ovarian primary tumors; however, no somatic mutations in EP300 have been identified in any primary tumor [40,41]. EP300 is a histone acetyltransferase that regulates transcription via chromatin remodeling, and plays an important role in the processes of cell proliferation and differentiation. EP300 acetylation of tumor protein P53 (TP53) in response to DNA damage regulates the DNA-binding and transcription functions of TP53 [42]. The tumor suppressor gene protein arginine methyltransferase 2 (PRMT2) inhibits nuclear factor of kappa light polypeptide gene enhancer in B-cells $1(\mathrm{NF}-\kappa \mathrm{B})$-dependent transcription and promotes apoptosis, by blocking nuclear export of nuclear factor of kappa light polypeptide gene enhancer in B-cells inhibitor, alpha $(\mathrm{I} \kappa \mathrm{B}-\alpha)$ via a leptomycin-sensitive pathway, which increases nuclear I $\mathrm{K}-\alpha$ accumulation and decreases NF- $\kappa$ B DNA binding [43-46]. The highly conserved $S$-adenosylmethionine-binding domain of PRMT2 mediates this effect. PRMT2 also renders cells susceptible to apoptosis induced by cytokines or cytotoxic drugs, most likely due to the effects of PRMT2 on NF- $\kappa B$. Mouse embryo fibroblasts from PRMT2 genetic knockouts have elevated NF- $\mathrm{BB}$ activity and decreased susceptibility to apoptosis, compared to wild-type or complemented cells. These data suggest that PRMT2 inhibits cell activation and promotes programmed cell death through a NF- $\mathrm{B}$-dependent mechanism. 
Table 3. Genes encoding epigenetic chromatin modification enzymes downregulated in normal karyotype B cell pediatric ALL compared with normal controls.

\begin{tabular}{lcccccc}
\hline & Gene & Description & NBM & ALL & Change & $\boldsymbol{p}$ value \\
\hline 1 & HDAC5 & Histone deacetylase 5 & 11379.83 & 186.94 & 0.01 & $2.67 \times 10^{-27}$ \\
2 & NOTCH2 & Notch homolog 2 & 32473.8 & 319.09 & 0.01 & $3.05 \times 10^{-27}$ \\
3 & NOTCH1 & Notch homolog 1 & 13109.41 & 1089.23 & 0.05 & $9.19 \times 10^{-15}$ \\
4 & EP300 & E1A binding protein p300 & 47487.19 & 5601.60 & 0.12 & $1.37 \times 10^{-13}$ \\
5 & PRMT2 & Protein arginine methyltransferase 2 & 28388.11 & 6336.82 & 0.22 & $2.08 \times 10^{-12}$ \\
6 & DNMT3A & DNA (cytosine) methyltransferase 3 alpha & 3868.084 & 682.15 & 0.17 & $2.04 \times 10^{-12}$ \\
7 & RPS6KA3 & Ribosomal protein S6 polypeptide 3 & 20389.11 & 5203.03 & 0.25 & $1.00 \times 10^{-5}$ \\
\hline
\end{tabular}

NBM, Normal Bone Marrow.

Figure 5. Expression of downregulated epigenetic chromatin modification genes in normal karyotype B cell pediatric ALL. Expression levels of the significantly downregulated genes in normal karyotype B cell pediatric ALL $(n=18)$, compared to the control samples $(\mathrm{NBT} / \mathrm{IPT} ; n=20)$. Data is presented as the average $\pm \mathrm{SE}$; $p$ values $<0.05$ were considered statistically significant.
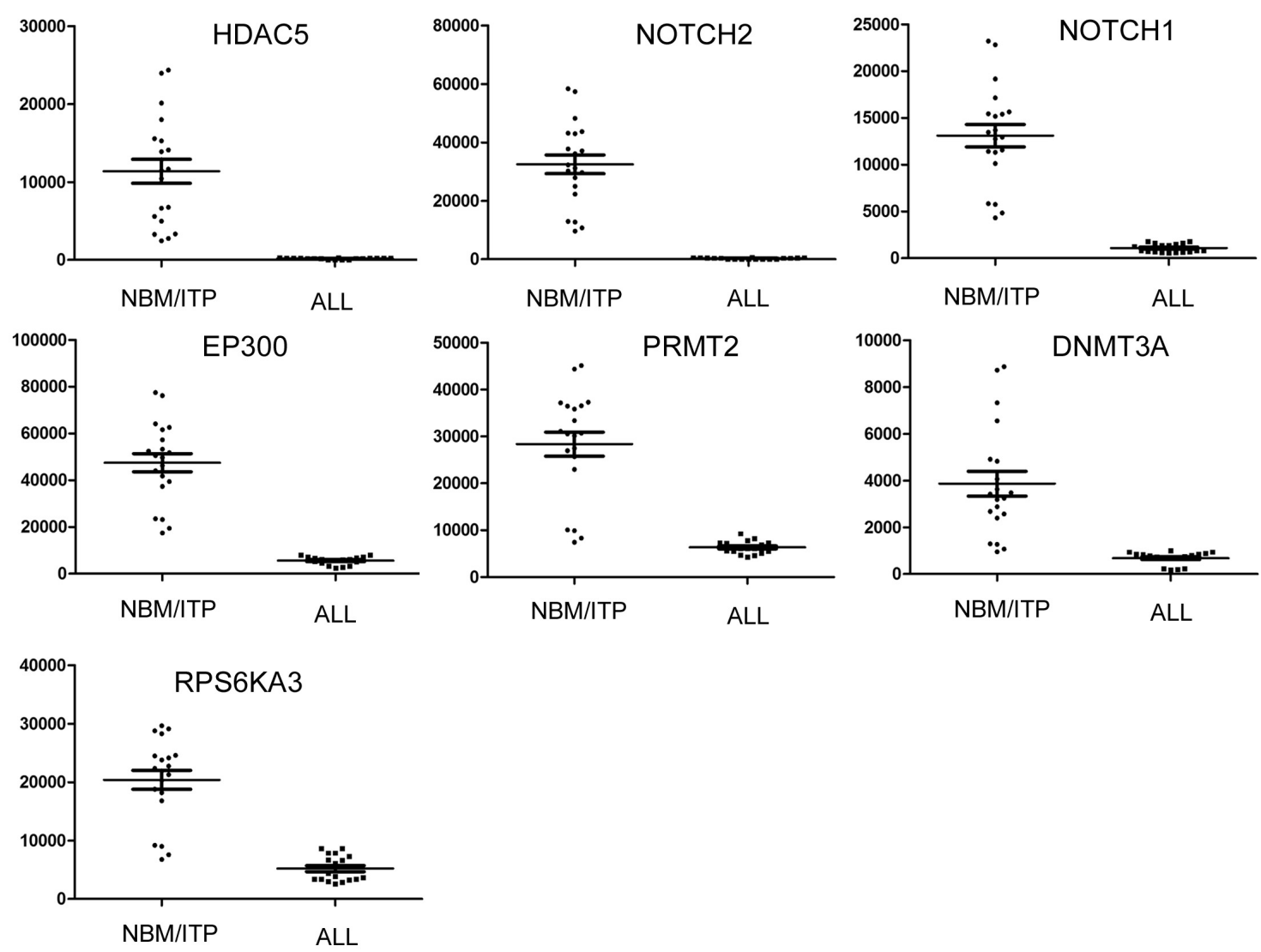

2.4. Ingenuity Pathway Analysis of Dys-regulated Genes in Normal Karyotype B cell Pediatric ALL

To investigate the possible biological interactions between the differently regulated genes in pediatric ALL, the datasets derived from the real-time PCR array analyses were imported into the Ingenuity Pathway Analysis (IPA) Tool. IPA analysis of the genes with a significantly altered expression profile in ALL revealed two significant networks (Figure 6A). Of these networks, Gene 
Expression and Organ Morphology was the highest rated network, with 13 focus molecules and a significance score of 35 (Figure 6D). The score is the probability that a collection of genes equal to or greater than the number of genes in the network could be achieved by chance alone. A score of 3 indicates a 1/1000 chance that the focus genes are not in the network due to random chance. The IPA analysis also grouped the differentially expressed genes in pediatric ALL into a number of other biological mechanisms related to Phospholipase C Signaling $\left(1.79 \times 10^{-4}\right)$, HMGB1 Signaling $\left(2.22 \times 10^{-4}\right)$, DNA methylation and Transcriptional Repression Signaling $\left(2.99 \times 10^{-4}\right)$, Hereditary Breast Cancer Signaling $\left(3.84 \times 10^{-4}\right)$ and Nocth Signaling $\left(1.03 \times 10^{-3}\right.$, Figure 6 B). Further results of the IPA analysis are provided in Supplementary File 3. IPA analysis of the significantly dys-regualted histone-modifying enzymes in pediatric ALL.

Figure 6. Summary of Ingenuity Pathway Analysis for dys-regulated epigenetic chromatin modification genes in normal karyotype B cell pediatric ALL. To investigate possible interactions between the differently regulated genes in pediatric ALL, datasets representing the 18 significantly altered genes were imported into the Ingenuity Pathway Analysis (IPA) Tool. (A) Top two networks obtained from IPA (with their respective scores) for the differently regulated genes in pediatric ALL; (B) Toxicology pathway list obtained from IPA analysis for the differently regulated genes in pediatric ALL. The $x$-axis represents the most significant toxicology functions based on the differentially expressed genes are highlighted; the $y$-axis represents the number of genes from the dataset that map to the pathway and the number of all known genes ascribed to the pathway. The yellow line represents the threshold $p$ value $(0.05)$, as calculated by Fisher's test; (C) Upstream regulator list for the differently regulated genes in pediatric ALL. Curcumin and mir-34 were the two most significant upstream regulators of the differently regulated genes in pediatric ALL; (D) Network representation of the most highly rated network for the differently regulated genes in pediatric ALL. The shaded genes are statistically significant. Solid lines represent a direct interaction between two gene products, dotted lines represent indirect interactions; (E) Mapping of the genes associated with the upstream regulators for the differently regulated genes in pediatric ALL.

A

\section{Top Networks}

Associated Network Functions

Gene Expression, Organ Morphology, Skeletal and Muscular System Development and Function

DNA Replication, Recombination, and Repair, Gene Expression, Cellular Function and Maintenance

Score

10

\section{B}

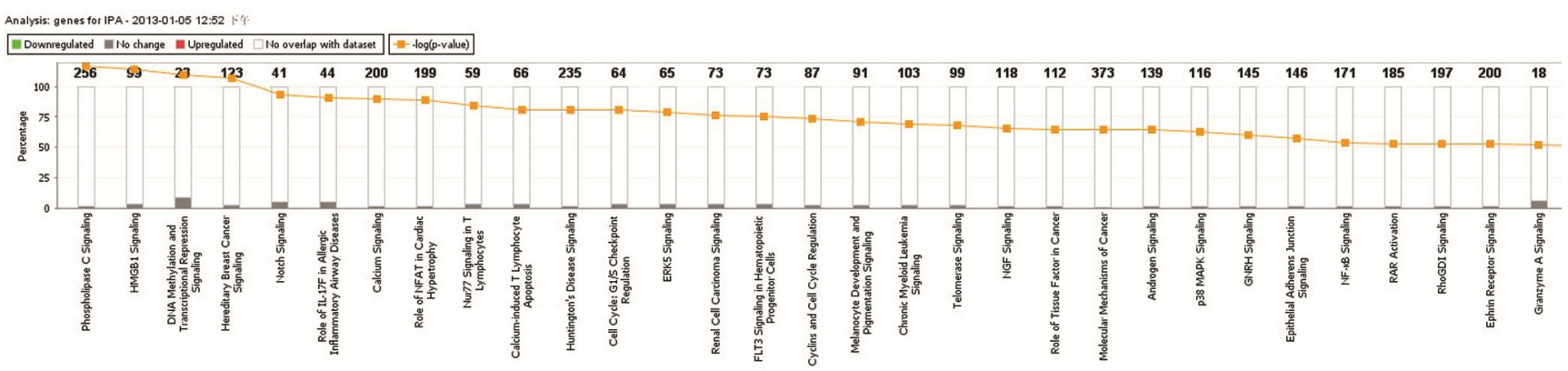


Figure 6. Cont.

C Top Upstream Regulators

\begin{tabular}{|c|c|c|}
\hline Upstream Regulator & $\mathrm{p}$-value of overlap & $\begin{array}{l}\text { Predicted } \\
\text { Activation } \\
\text { State }\end{array}$ \\
\hline curcumin & $2.83 E-06$ & \\
\hline mir-34 & $2.45 \mathrm{E}-05$ & \\
\hline miR-34a-5p (and other miRNAs wiseed GGCAGUG) & 1.33E-04 & \\
\hline POFUT1 & $9.63 \mathrm{E}-04$ & \\
\hline PCNA & $9.63 \mathrm{E}-04$ & \\
\hline
\end{tabular}

$\mathrm{D}$

$E$
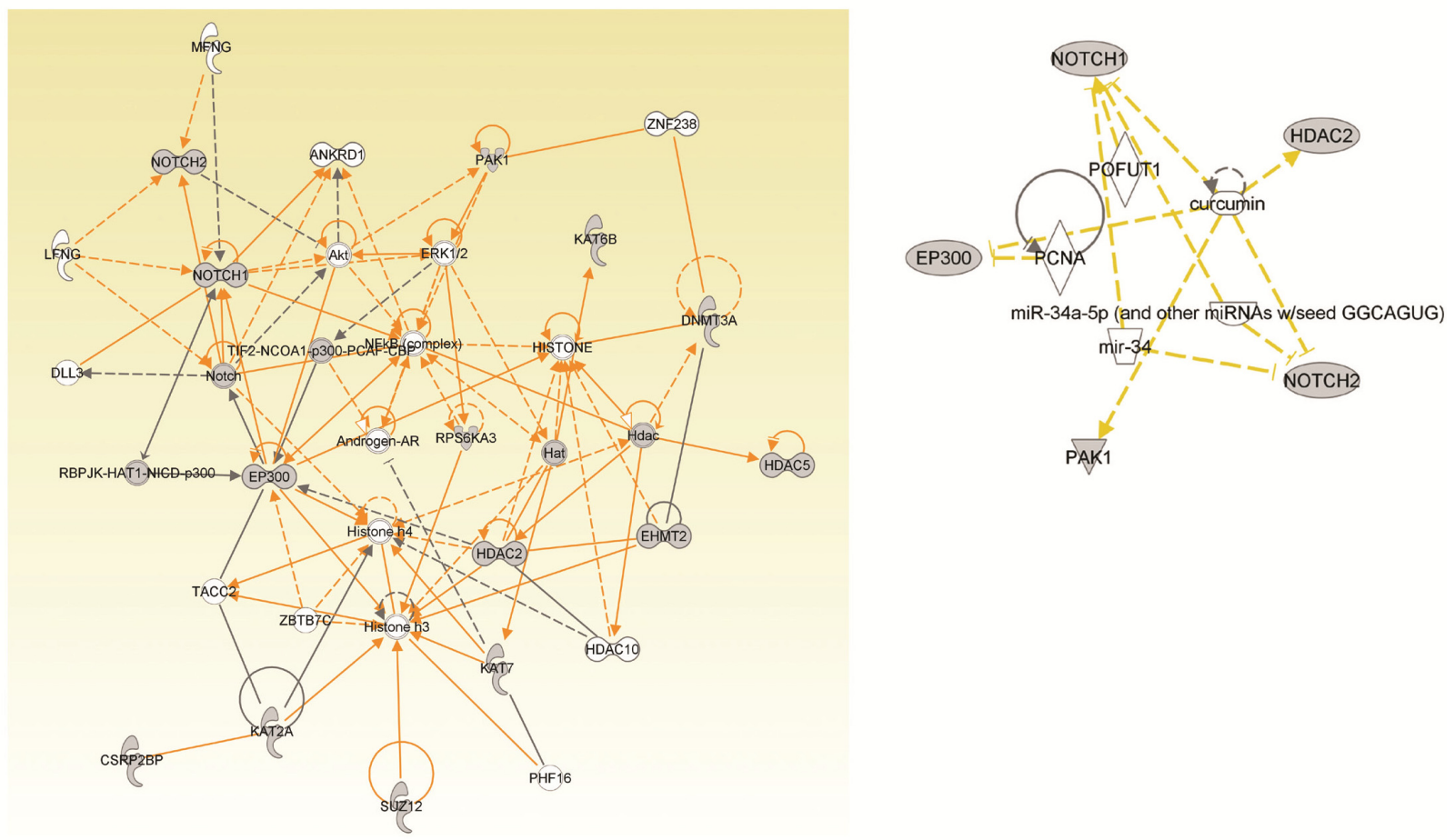

The IPA analysis also revealed that curcumin and mir-34 signaling were the two most important upstream regulators for the dysregulated_histone-modifying enzymes in pediatric ALL, with $p$ values of $2.83 \times 10^{-6}$ and $2.45 \times 10^{-5}$, respectively (Figure 6C). The genes associated with the upstream regulators are mapped in Figure 4E. Ectopic expression of miR-34 genes leads to marked effects on cell proliferation and survival, due to cell-cycle arrest in the G1 phase [47,48]. Interestingly, the introduction of miR-34a and miR-34b/c induced cellular senescence in primary human diploid fibroblasts, and overexpression of miR-34a induced apoptosis in tumor cells. MiR-34a has been shown to target and translationally repress sirtuin 1 (SIRT1) mRNA [49-51]. SIRT1, a histone-modifying enzyme, is a NAD-dependent deacetylase which has been shown to inhibit the activity of several pro-apoptotic proteins. Regulation of SIRT1 by miR-34a forms part of a positive feedback loop which leads to enhanced activation of $\mathrm{p} 53$, once it has been initially activated. This study provides the first indication that other histone-modifying enzymes, in addition to SIRT1, may be dys-regulated by miR-34 in pediatric ALL.

The other upstream regulator of histone-modifying enzymes in normal karyotype B cell pediatric ALL revealed in this study was curcumin (diferuloylmethane), which is a polyphenol derived from the 
plant Curcuma longa, commonly known as turmeric. Recently, curcumin has been found to possess anti-cancer activity, as it exerts a number of effects on a variety of biological pathways involved in mutagenesis, oncogene expression, cell cycle regulation, apoptosis, tumorigenesis and metastasis. Curcumin has demonstrated anti-proliferative effects in multiple types of cancer, and is an inhibitor of the transcription factor NF- $\mathrm{BB}$ and its downstream gene products including $c-M Y C, B C L-2, C O X-2$, $N O S$, Cyclin D1, TNF- $\alpha$, interleukins and MMP-9. In addition, curcumin affects a variety of growth factor receptors and cell adhesion molecules involved in tumor growth, angiogenesis and metastasis. Cultured leukemia cells are particularly responsive to curcumin [52-54]. As of 2011, more than 75 studies in peer-reviewed journals have reported that curcumin induces apoptosis and cell death in cultured animal and human leukemia cells. This study is the first to imply that curcumin may affect cancer cell growth and apoptosis via regulation of histone-modifying enzymes. In the future, we will seek to validate these results, and examine the role of curcumin and miR-34 in the molecular basis of leukemia.

\section{Experimental Section}

\subsection{Patients and Samples}

Bone marrow specimens were obtained at the time of diagnosis during routine clinical assessment of 30 patients with ALL, who presented at the Department of Hematology and Oncology, Children's Hospital of Soochow University between 2010 and 2012. Ethical approval was provided by the Children's Hospital of Soochow University Ethics Committee (No.SUEC2010-011 and No. SUEC2009-219-1), and informed consent was obtained from the parents or guardians. The main clinical and laboratory features of the patient cohort are summarized in Table 1. Additionally, bone marrow samples from 10 healthy donors from surgical operations and 10 patients with idiopathic thrombocytopenic purpura (ITP) were analyzed as controls. Bone marrow mononuclear cells (BMNCs) were isolated using Ficoll solution within $2 \mathrm{~h}$ after harvest.

\subsection{RNA Extraction}

BMNCs were immediately submerged in $4 \mathrm{~mL}$ TRIzol (Invitrogen, Carlsbad, CA, USA), and stored at $-80{ }^{\circ} \mathrm{C}$ until further processing. A volume of $1.2 \mathrm{~mL}$ from each sample was centrifuged at $12,000 \mathrm{~g}$ for $15 \mathrm{~min}$ at $4{ }^{\circ} \mathrm{C}$ to remove debris and DNA, then $1 \mathrm{~mL}$ of the supernatant was mixed with $200 \mu \mathrm{L}$ chloroform, shaken for 15 seconds, incubated at RT for 2-3 min and centrifuged at 12,000 $\mathrm{g}$ for $10 \mathrm{~min}$ at $4{ }^{\circ} \mathrm{C}$. RNA was precipitated by adding $500 \mu \mathrm{L}$ of the aqueous phase to an equal volume of isopropanol and centrifugation at $14,000 \mathrm{~g}$ for $10 \mathrm{~min}$ at $4{ }^{\circ} \mathrm{C}$. The RNA pellet was washed with $75 \%$ ethanol, centrifuged at $14,000 \mathrm{~g}$ for $10 \mathrm{~min}$ at $4{ }^{\circ} \mathrm{C}$, dried and resuspended in $60 \mu \mathrm{L}$ DEPC-treated $\mathrm{H}_{2} \mathrm{O}$. The final RNA concentration of the samples was determined using a spectrophotometer (Nanodrop 2000, Thermo-scientific, Wilmington, DE, USA) and the purity of the RNA samples was assessed by agarose gel electrophoresis. 


\subsection{Synthesis of cDNA}

Synthesis of cDNA was performed using $4 \mu \mathrm{g}$ of RNA in $10 \mu \mathrm{L}$ reactions with SuperScript II reverse transcriptase (Invitrogen, Carlsbad, CA, USA), as recommended by the manufacturer. The RNA was incubated with $0.5 \mu \mathrm{g}$ of oligo(dT)12-18mers primers (Invitrogen) for $7 \mathrm{~min}$ at $70{ }^{\circ} \mathrm{C}$ and then transferred onto ice. Then, $9 \mu \mathrm{L}$ of a master mix containing $4 \mu \mathrm{L}$ of SuperScript II buffer, $2 \mu \mathrm{L}$ of $0.1 \mathrm{M}$ DTT (Invitrogen), and $1 \mu \mathrm{L}$ each of dNTPs (10 mM; Invitrogen), RNasin (40 UI; Promega, Madison, WI, USA) and SuperScript II (Invitrogen) were added, centrifuged and incubated at $42{ }^{\circ} \mathrm{C}$ for $60 \mathrm{~min}$, followed by $5 \mathrm{~min}$ at $70{ }^{\circ} \mathrm{C}$ to inactivate the enzyme; the cDNA was stored at $-20{ }^{\circ} \mathrm{C}$.

\subsection{Real-Time PCR Array Design and Testing}

Most of the primers were obtained from the database of real-time primers curated by the Center for Medical Genetics (http://medgen.ugent.be/CMGG/). The remainders of the primers were designed using the online program Primer 3 (www.fokker.wi.mit.edu/primer3/input.htm). The primer selection parameters were primer size: $20-26 \mathrm{nts}$; primer melting temperature: $60^{\circ} \mathrm{C}$ to $64{ }^{\circ} \mathrm{C}$; GC clamp: 1 ; and product size range: generally $120-240 \mathrm{bp}$, but reduced to $100 \mathrm{bp}$ if no appropriate primers could be identified. The sequences of the primers are listed in Supplementary File 1. All of the primers were synthesized by Invitrogen.

\subsection{Real-Time PCR Array Analysis}

Real-time PCR array analysis was performed in a total volume of $20 \mu \mathrm{L}$ including $2 \mu \mathrm{L}$ of cDNA, primers (0.2 $\mathrm{mM}$ each) and $10 \mu \mathrm{L}$ of SYBR Green mix (Roche, Basel, Switzerland). Reactions were run on an Light cycler 480 (Roche, Basel, Switzerland) using universal thermal cycling parameters $\left(95{ }^{\circ} \mathrm{C}\right.$ for $5 \mathrm{~min}, 45$ cycles of $10 \mathrm{~s}$ at $95{ }^{\circ} \mathrm{C}, 20 \mathrm{~s}$ at $60{ }^{\circ} \mathrm{C}$ and $15 \mathrm{~s}$ at $72{ }^{\circ} \mathrm{C}$; followed by a melting curve: $10 \mathrm{~s}$ at $95{ }^{\circ} \mathrm{C}, 60 \mathrm{~s}$ at $60{ }^{\circ} \mathrm{C}$ and continued melting). The results were obtained using the sequence detection software of the Light cycler 480 and analyzed using Microsoft Excel. For quality control purposes, melting curves were acquired for all samples. The comparative $C_{t}$ method was used to quantify gene expression. Firstly, the target gene expression level was normalized to expression of the housekeeping gene glyceraldehyde 3-phosphate dehydrogenase $(G A P D H)$ within the same sample $(-\Delta \mathrm{Ct})$, and then the relative expression of each gene was calculated using $10^{6} \times \log _{2}(-\Delta \mathrm{Ct})$. The gene expression of the pediatric ALL and control samples was presented as the average $\pm \mathrm{SE}$.

\subsection{Western Blot Analysis}

For western blot analysis, cellular proteins were extracted in $40 \mathrm{mM}$ Tris- $\mathrm{HCl}(\mathrm{pH} 7.4)$ containing $150 \mathrm{mM} \mathrm{NaCl}$ and $1 \%(v / v)$ Triton X-100, supplemented with a cocktail of protease inhibitors. Equal amounts of protein were resolved on 12\% SDS-PAGE gels, and then transferred to a PVDF membrane (Millipore, Bedford, MA, USA). Blots were blocked and then probed with antibodies against PAK1 (1:1000, Cell Signaling Technology, Inc., Danvers, MA, USA), HDAC2 (1:1000, Santa Cruz Biotechnology, Inc., Santa Cruz, CA, USA), GAPDH (1:5000, Sigma, St. Louis, MO, USA). After washing, the blots were incubated with horseradish peroxidase-conjugated secondary antibodies and 
visualized by enhanced chemiluminescence kit (Pierce, Rockford, IL, USA). Protein bands were visualized after exposure of the membrane to Kodak X-ray film.

\subsection{Ingenuity Pathway Analysis (IPA)}

Datasets derived from the real-time PCR array analyses, representing genes with significantly altered expression profiles, were imported into the Ingenuity Pathway Analysis Tool (IPA Tool; Ingenuity H Systems, Redwood City, CA, USA; http://www.ingenuity.com). In IPA, differentially expressed genes are mapped to the genetic networks available in the Ingenuity database, and then ranked by score. The Ingenuity Pathway Knowledge Base (IPKB) forms the basis of IPA, and is derived from known gene functions and interactions published in the literature. Thus, IPA enables the identification of biological networks, global functions and functional pathways for a particular dataset. The program also calculates the significance of the genes in the network, the other genes with which it interacts, and how the products of the genes directly or indirectly act on each other, including those not involved in the microarray analysis. The networks created are ranked, depending on the number of significantly expressed genes they contain, and also the list of significant, relevant diseases. A network is a graphical representation of the molecular relationships between molecules. Molecules are represented as nodes, and the biological relationship between two nodes is represented as an edge (line). All edges are supported by at least one reference from the literature, a textbook, or canonical information stored in the Ingenuity Pathways Knowledge Base. The intensity of the node color indicates the degree of upregulation (red) or downregulation (green). Nodes are displayed using various shapes that represent the functional class of the gene product.

\subsection{Statistical Analysis}

The significance of the differences in the gene expression profiles of pediatric ALL and the control samples were calculated using unpaired $t$-tests with SPSS version 11.5 (SPSS Inc., Chicago, IL, USA); $p$ values $<0.05$ were considered statistically significant.

\section{Conclusions}

We successfully designed and tested a real-time PCR array for analysis of the genes encoding human epigenetic chromatin modification enzymes. Using this array, we demonstrated the different mRNA expression patterns of human histone-modifying enzymes in normal karyotype B cell pediatric ALL and normal controls. A lot of genes can be significantly clustered in the gene and sample analysis, including the histone deacetylases $H D A C 2$ which was upregulated in normal karyotype B cell pediatric ALL, PRMT2 and the putative tumor suppressor gene EP300 which were downregulated in pediatric ALL. We identified a number of dysregulated histone-modifying enzymes in normal karyotype B cell pediatric ALL, which have not previously been reported to be differently expressed in pediatric ALL. Future studies will seek to determine whether these dys-regulated histone-modifying enzymes can serve as biomarkers of pediatric ALL. Additionally, IPA indicated that curcumin and miR-34 may be the major upstream regulators of histone-modifying enzymes in normal karyotype B cell pediatric ALL, future studies will seek to validate these results, and examine the role of curcumin 
and miR-34 in the molecular basis of leukemia. This work provides new clues regarding the molecular mechanisms which regulate the development of normal karyotype B cell pediatric ALL.

\section{Acknowledgments}

This work was supported by grants from the National Key Basic Research Program (No. 2010CB933902), the National Natural Science Foundation for Youth (No. 81100371), the Natural Science Foundation of Jiangsu Province (No. BK2011308), the Universities Natural Science Foundation of Jiangsu Province (No. 11KJB320014), the Talent's Subsidy project in Science and Education of Department of Public Health of Suzhou City (No. SWKQ1020), the Medical Innovation Team and Leading Talent of Jiangsu Province (No. LJ201126), the Major Scientific and Technological Special Project for “Significant New Drugs Creation” (No. 2012ZX09103301-040).

\section{Conflicts of Interest}

The authors declare no conflict of interest.

\section{References}

1. Foa, R. Acute lymphoblastic leukemia: Age and biology. Pediatr. Rep. 2011, 3, e2.

2. Krupa, M.; Szczepanski, T. Prophylaxis of hepatitis B in children treated for ALL. Wiad. Lek. 2009, 62, 149-152.

3. Pui, C.H. Acute lymphoblastic leukemia: Introduction. Semin. Hematol. 2009, 46, 1-2.

4. Salek, C.; Sponerova, D.; Soukupova Maaloufova, J. Acute lymphoblastic leukemia: Past and present. Vnitr. Lek. 2012, 58, 20-26.

5. Zangrando, A.; Dell'orto, M.C.; Te Kronnie, G.; Basso, G. MLL rearrangements in pediatric acute lymphoblastic and myeloblastic leukemias: MLL specific and lineage specific signatures. BMC Med. Genomics 2009, 2, 36.

6. Mishra, B.P.; Ansari, K.I.; Mandal, S.S. Dynamic association of MLL1, H3K4 trimethylation with chromatin and Hox gene expression during the cell cycle. FEBS J. 2009, 276, 1629-1640.

7. Passaro, D.; Rana, G.; Piscopo, M.; Viggiano, E.; de Luca, B.; Fucci, L. Epigenetic chromatin modifications in the cortical spreading depression. Brain Res. 2010, 1329, 1-9.

8. Krivtsov, A.V.; Armstrong, S.A. MLL translocations, histone modifications and leukaemia stem-cell development. Nat. Rev. Cancer 2007, 7, 823-833.

9. Cosgrove, M.S.; Patel, A. Mixed lineage leukemia: A structure-function perspective of the MLL1 protein. FEBS J. 2010, 277, 1832-1842.

10. Islam, A.B.; Richter, W.F.; Jacobs, L.A.; Lopez-Bigas, N.; Benevolenskaya, E.V. Co-regulation of histone-modifying enzymes in cancer. PLoS One 2011, 6, e24023.

11. Fullgrabe, J.; Kavanagh, E.; Joseph, B. Histone onco-modifications. Oncogene 2011, 30, 3391-3403.

12. Henrique, R.; Luis, A.S.; Jeronimo, C. The epigenetics of renal cell tumors: From biology to biomarkers. Front. Genet. 2012, 3, 94.

13. Nemeth, A.; Langst, G. Chromatin higher order structure: Opening up chromatin for transcription. Brief Funct. Genomic Proteomic 2004, 2, 334-343. 
14. Zhang, Y.; Moriguchi, H. Chromatin remodeling system, cancer stem-like attractors, and cellular reprogramming. Cell. Mol. Life Sci 2011, 68, 3557-3571.

15. Del Rizzo, P.A.; Trievel, R.C. Substrate and product specificities of SET domain methyltransferases. Epigenetics 2011, 6, 1059-1067.

16. Albaugh, B.N.; Arnold, K.M.; Denu, J.M. KAT(ching) metabolism by the tail: Insight into the links between lysine acetyltransferases and metabolism. Chembiochem 2011, 12, 290-298.

17. Hou, H.; Yu, H. Structural insights into histone lysine demethylation. Curr. Opin. Struct. Biol. 2010, 20, 739-748.

18. Krichevsky, A.; Zaltsman, A.; Lacroix, B.; Citovsky, V. Involvement of KDM1C histone demethylase-OTLD1 otubain-like histone deubiquitinase complexes in plant gene repression. Proc. Natl. Acad. Sci. USA 2011, 108, 11157-11162.

19. Luo, X.; Liu, Y.; Kubicek, S.; Myllyharju, J.; Tumber, A.; Ng, S.; Che, K.H.; Podoll, J.; Heightman, T.D.; Oppermann, U.; et al. A selective inhibitor and probe of the cellular functions of Jumonji C domain-containing histone demethylases. J. Am. Chem. Soc. 2011, 133, 9451-9456.

20. Marmorstein, R.; Trievel, R.C. Histone modifying enzymes: Structures, mechanisms, and specificities. Biochim. Biophys. Acta 2009, 1789, 58-68.

21. Upadhyay, A.K.; Cheng, X. Dynamics of histone lysine methylation: Structures of methyl writers and erasers. Prog. Drug Res. 2011, 67, 107-124.

22. Lawless, M.W.; Norris, S.; O’Byrne, K.J.; Gray, S.G. Targeting histone deacetylases for the treatment of disease. J. Cell. Mol. Med. 2009, 13, 826-852.

23. Tao, Y.F.; Wu, D.; Pang, L.; Zhao, W.L.; Lu, J.; Wang, N.; Wang, J.; Feng, X.; Li, Y.H.; Ni, J.; et al. Analyzing the gene expression profile of pediatric acute myeloid leukemia with real-time PCR arrays. Cancer Cell. Int. 2012, 12, 40.

24. Arikawa, E.; Sun, Y.; Wang, J.; Zhou, Q.; Ning, B.; Dial, S.L.; Guo, L.; Yang, J. Cross-platform comparison of SYBR Green real-time PCR with TaqMan PCR, microarrays and other gene expression measurement technologies evaluated in the MicroArray Quality Control (MAQC) study. BMC Genomics 2008, 9, 328.

25. Moreno, D.A.; Scrideli, C.A.; Cortez, M.A.; de Paula Queiroz, R.; Valera, E.T.; da Silva Silveira, V.; Yunes, J.A.; Brandalise, S.R.; Tone, L.G. Differential expression of HDAC3, HDAC7 and HDAC9 is associated with prognosis and survival in childhood acute lymphoblastic leukaemia. Br. J. Haematol. 2010, 150, 665-673.

26. Niegisch, G.; Knievel, J.; Koch, A.; Hader, C.; Fischer, U.; Albers, P.; Schulz, W.A. Changes in histone deacetylase (HDAC) expression patterns and activity of HDAC inhibitors in urothelial cancers. Urol. Oncol. 2012, [Epub ahead of print].

27. Patani, N.; Jiang, W.G.; Newbold, R.F.; Mokbel, K. Histone-modifier gene expression profiles are associated with pathological and clinical outcomes in human breast cancer. Anticancer Res. 2011, $31,4115-4125$.

28. Noh, J.H.; Jung, K.H.; Kim, J.K.; Eun, J.W.; Bae, H.J.; Xie, H.J.; Chang, Y.G.; Kim, M.G.; Park, W.S.; Lee, J.Y.; et al. Aberrant regulation of HDAC2 mediates proliferation of hepatocellular carcinoma cells by deregulating expression of $\mathrm{G} 1 / \mathrm{S}$ cell cycle proteins. PLoS One 2011, 6, e28103. 
29. Zhu, P.; Martin, E.; Mengwasser, J.; Schlag, P.; Janssen, K.P.; Gottlicher, M. Induction of HDAC2 expression upon loss of APC in colorectal tumorigenesis. Cancer Cell 2004, 5, 455-463.

30. Zhu, G.; Wang, Y.; Huang, B.; Liang, J.; Ding, Y.; Xu, A.; Wu, W. A Rac1/PAK1 cascade controls beta-catenin activation in colon cancer cells. Oncogene 2012, 31, 1001-1012.

31. Lee, M.Y.; Kim, S.H.; Ihm, H.J.; Chae, H.D.; Kim, C.H.; Kang, B.M. Up-regulation of p21-activated kinase 1 by in vitro treatment with interleukin 1-beta and its increased expression in ovarian endometriotic cysts. Fertil Steril 2011, 96, 508-511.

32. Kamai, T.; Shirataki, H.; Nakanishi, K.; Furuya, N.; Kambara, T.; Abe, H.; Oyama, T.; Yoshida, K. Increased Rac1 activity and Pak1 overexpression are associated with lymphovascular invasion and lymph node metastasis of upper urinary tract cancer. BMC Cancer 2010, 10, 164.

33. Kim, S.R.; Kim, S.H.; Lee, H.W.; Chae, H.D.; Kim, C.H.; Kang, B.M. Increased expression of p21-activated kinase in adenomyosis. Fertil Steril 2010, 94, 1125-1128.

34. Wang, R.A.; Vadlamudi, R.K.; Bagheri-Yarmand, R.; Beuvink, I.; Hynes, N.E.; Kumar, R. Essential functions of $\mathrm{p} 21$-activated kinase 1 in morphogenesis and differentiation of mammary glands. J. Cell. Biol. 2003, 161, 583-592.

35. Akinmade, D.; Talukder, A.H.; Zhang, Y.; Luo, W.M.; Kumar, R.; Hamburger, A.W. Phosphorylation of the ErbB3 binding protein Ebp1 by p21-activated kinase 1 in breast cancer cells. Br. J. Cancer 2008, 98, 1132-1140.

36. Siu, M.K.; Wong, E.S.; Chan, H.Y.; Kong, D.S.; Woo, N.W.; Tam, K.F.; Ngan, H.Y.; Chan, Q.K.; Chan, D.C.; Chan, K.Y.; et al. Differential expression and phosphorylation of Pak1 and Pak2 in ovarian cancer: Effects on prognosis and cell invasion. Int. J. Cancer 2010, 127, 21-31.

37. Tharakan, R.; Lepont, P.; Singleton, D.; Kumar, R.; Khan, S. Phosphorylation of estrogen receptor alpha, serine residue 305 enhances activity. Mol. Cell. Endocrinol. 2008, 295, 70-78.

38. Wang, J.X.; Zhou, Y.N.; Zou, S.J.; Ren, T.W.; Zhang, Z.Y. Correlations of P21-activated kinase 1 expression to clinicopathological features of gastric carcinoma and patients' prognosis. Chin. J. Cancer 2010, 29, 649-654.

39. Liu, F.; Li, X.; Wang, C.; Cai, X.; Du, Z.; Xu, H.; Li, F. Downregulation of p21-activated kinase-1 inhibits the growth of gastric cancer cells involving cyclin B1. Int. J. Cancer 2009, 125, 2511-2519.

40. Tillinghast, G.W.; Partee, J.; Albert, P.; Kelley, J.M.; Burtow, K.H.; Kelly, K. Analysis of genetic stability at the EP300 and CREBBP loci in a panel of cancer cell lines. Genes Chromosomes Cancer 2003, 37, 121-131.

41. Bryan, E.J.; Jokubaitis, V.J.; Chamberlain, N.L.; Baxter, S.W.; Dawson, E.; Choong, D.Y.; Campbell, I.G. Mutation analysis of EP300 in colon, breast and ovarian carcinomas. Int. J. Cancer 2002, 102, 137-141.

42. Gayther, S.A.; Batley, S.J.; Linger, L.; Bannister, A.; Thorpe, K.; Chin, S.F.; Daigo, Y.; Russell, P.; Wilson, A.; Sowter, H.M.; et al. Mutations truncating the EP300 acetylase in human cancers. Nat. Genet. 2000, 24, 300-303.

43. Zhong, J.; Cao, R.X.; Zu, X.Y.; Hong, T.; Yang, J.; Liu, L.; Xiao, X.H.; Ding, W.J.; Zhao, Q.; Liu, J.H.; et al. Identification and characterization of novel spliced variants of PRMT2 in breast carcinoma. FEBS J. 2012, 279, 316-335. 
44. Hata, K.; Nishijima, K.; Mizuguchi, J. Role for Btg1 and Btg2 in growth arrest of WEHI-231 cells through arginine methylation following membrane immunoglobulin engagement. Exp. Cell. Res. 2007, 313, 2356-2366.

45. Scoumanne, A.; Chen, X. The epithelial cell transforming sequence 2, a guanine nucleotide exchange factor for Rho GTPases, is repressed by p53 via protein methyltransferases and is required for G1-S transition. Cancer Res. 2006, 66, 6271-6279.

46. Ganesh, L.; Yoshimoto, T.; Moorthy, N.C.; Akahata, W.; Boehm, M.; Nabel, E.G.; Nabel, G.J. Protein methyltransferase 2 inhibits NF-kappaB function and promotes apoptosis. Mol. Cell. Biol. 2006, 26, 3864-3874.

47. Wong, K.Y.; Yu, L.; Chim, C.S. DNA methylation of tumor suppressor miRNA genes: A lesson from the miR-34 family. Epigenomics 2011, 3, 83-92.

48. Hermeking, H. The miR-34 family in cancer and apoptosis. Cell Death Differ. 2010, 17, 193-199.

49. Tabuchi, T.; Satoh, M.; Itoh, T.; Nakamura, M. MicroRNA-34a regulates the longevity-associated protein SIRT1 in coronary artery disease: Effect of statins on SIRT1 and microRNA-34a expression. Clin. Sci. 2012, 123, 161-171.

50. Yamakuchi, M.; Lowenstein, C.J. MiR-34, SIRT1 and p53: The feedback loop. Cell Cycle 2009, 8, 712-715.

51. Yamakuchi, M.; Ferlito, M.; Lowenstein, C.J. miR-34a repression of SIRT1 regulates apoptosis. Proc. Natl. Acad. Sci. USA 2008, 105, 13421-13426.

52. Shishodia, S. Molecular mechanisms of curcumin action: Gene expression. Biofactors 2012, doi:10.1002/biof.1041.

53. Basnet, P.; Skalko-Basnet, N. Curcumin: An anti-inflammatory molecule from a curry spice on the path to cancer treatment. Molecules 2011, 16, 4567-4598.

54. Ravindran, J.; Prasad, S.; Aggarwal, B.B. Curcumin and cancer cells: How many ways can curry kill tumor cells selectively? AAPS J. 2009, 11, 495-510.

(C) 2013 by the authors; licensee MDPI, Basel, Switzerland. This article is an open access article distributed under the terms and conditions of the Creative Commons Attribution license (http://creativecommons.org/licenses/by/3.0/). 